\title{
Effect of microRNA-133a-3p/matrix metalloproteinase-9 axis on the growth of atherosclerotic vascular smooth muscle cells
}

\author{
LEI SHI ${ }^{1}$, CHUNPENG YU ${ }^{2}$, XINTAO TIAN ${ }^{1}$, CHENGTAI MA ${ }^{1}$, LUMIN WANG $^{1}$, DI XIA ${ }^{1}$, CHANGXING CUI $^{1}$, \\ XIAOXUE CHEN $^{1}$, TAO JIANG ${ }^{1}$, YAN GU $^{1}$, ZHENFANG LIU $^{1}$ and SHANGLANG CAI ${ }^{3}$ \\ Departments of ${ }^{1}$ Emergency Internal Medicine and ${ }^{2}$ Interventional Medicine, The Affiliated Hospital \\ of Qingdao University, Qingdao, Shandong 266071; ${ }^{3}$ Department of Cardiovascular Medicine, \\ The Affiliated Hospital of Qingdao University, Qingdao, Shandong 266000, P.R. China
}

Received November 21, 2018; Accepted August 1, 2019

DOI: $10.3892 / \mathrm{etm} .2019 .8070$

\begin{abstract}
Atherosclerosis (AS) is the leading cause of cardiovascular disease and poses a threat to human health. MicroRNAs (miRNAs/miRs) are a group of endogenous small non-coding RNAs that have been identified to serve important roles in AS. However, the expression and role of miR-133a-3p in AS remains unclear. The aim of the present study was to investigate miR-133a-3p in AS and to determine its underlying mechanism. The level of miR-133a-3p expression in the blood and vascular plaque tissue of patients with AS was detected via reverse transcription-quantitative PCR (RT-qPCR). The role of miR-133a-3p in human vascular smooth muscle cells (hVSMCs) was investigated, following upregulation and downregulation of this miR in hVSMCs. Cell proliferation and apoptosis were determined using a Cell Counting kit- 8 assay and flow cytometry, respectively. The results demonstrated the downregulation of miR-133a-3p in the blood and vascular plaque tissue of patients with AS. Matrix metallopeptidase-9 (MMP-9) was revealed to be a direct target gene of miR-133a-3p, which was upregulated in the blood and vascular plaque tissue of patients with AS. Furthermore, MMP-9 was determined to be negatively regulated by miR-133a-3p in hVSMCs. In addition, significant inhibition of hVSMC proliferation and induction of cell apoptosis were observed following MMP-9 downregulation and following transfection with the miR-133a-3p mimic. The effects of the miR-133a-3p mimic on hVSMC proliferation and apoptosis were reversed by MMP-9 over-expression. Overall, the results indicated that miR-133a-3p was downregulated in AS, which results in the inhibition of hVSMC proliferation and the induction of
\end{abstract}

Correspondence to: Dr Shanglang Cai, Department of Cardiovascular Medicine, The Affiliated Hospital of Qingdao University, 16 Jiangsu Road, Qingdao, Shandong 266000, P.R. China E-mail: cais1201717@163.com

Key words: miR-133a-3p, matrix metalloproteinase-9, atherosclerosis, vascular smooth muscle cells, proliferation cell apoptosis via MMP-9. miR-133a-3p may therefore be a promising therapeutic target for the treatment of AS.

\section{Introduction}

Atherosclerosis (AS) is a chronic inflammatory disease that is characterized by the accumulation of lipids and fibrous components in the aorta, which is the pathological basis for the development of cardiovascular disease $(1,2)$. Endothelial cell dysfunction (ECs) leads to the deposition of cholesterol and other lipids in the blood vessel wall, which is the initial stage of AS development (3). EC injury also leads to the intimal invasion of pro-inflammatory factors and the abnormal proliferation of vascular smooth muscle cells (VSMCs). The abnormal proliferation and migration of VSMCs are the main cause of local stenosis and AS lesions $(4,5)$. Therefore, the proliferation and migration of VSMCs serve a crucial role in the formation and development of AS plaques.

MicroRNAs (miRNAs/miRs) are a class of small non-coding RNAs comprised of 18-23 nucleotides that regulate gene expression by binding to the 3'-untranslated region (UTR) of target mRNAs (6). miRNAs have been identified to be involved in a variety of biological processes, including cellular differentiation, proliferation and apoptosis (7-9). Several studies have demonstrated the important roles that miRNAs serve in the development of AS. miR-365b-3p is involved in AS through the inhibition of human coronary artery smooth muscle cell proliferation and migration, by directly targeting a disintegrin and metalloproteinase within thrombospondin motifs 1 (10). Furthermore, miR-328 relieves oxidized low-density lipoprotein-induced EC injury by targeting high mobility group box 1 (11). miR-124-3p was also considered to be a risk factor for atherosclerotic disease (12) and miR-125b was revealed to inhibit VSMC proliferation and migration and to promote cell apoptosis (13).

miR-133a-3p has been well-studied in various types of cancer, including oropharyngeal (14), prostate (15), epithelial ovarian (16) and bladder cancer (17). Several studies have also demonstrated the critical roles served by miR-133a-3p in the regulation of cell proliferation and apoptosis (14-17). However, the expression and role of miR-133a-3p in AS remain unclear. 
Matrix metalloproteinase-9 (MMP-9) is a member of the endopeptidase family and is involved in the degradation and remodeling of the extracellular matrix (18). Enhanced MMP-9 expression in vascular component cells during vascular injury and inflammation serves an important role in the formation and rupture of atherosclerotic plaques (19). MMP-9 has also been reported to serve an important role in the regulation of VSMC proliferation and migration (20). However, the association between miR-133a-3p and MMP-9 remains unclear.

Therefore, the present study aimed to investigate the expression of miR-133a-3p in patients with AS, and to explore its role and molecular mechanism in the regulation of human (h)VSMC function.

\section{Materials and methods}

Clinical samples. Blood specimens, vascular plaque tissues and corresponding vascular tissues were obtained from 30 patients with AS (age range, 45-57 years; male/female, 15/15), and blood specimens were obtained from 30 healthy individuals (age range, 43-56 years; male/female: 15/15). The samples were obtained from the Affiliated Hospital of Qingdao University (Qingdao, China) between February 2016 and February 2018. Vascular plaque tissues and corresponding vascular tissues were obtained from patients with AS following coronary artery bypass surgery. Venous peripheral blood was drawn into heparin tubes from the 30 AS patients before surgery and from the 30 healthy individuals. Patients with the following conditions were excluded: Chronic or acute inflammatory disease, asthma, type I diabetes mellitus, autoimmune disease, cancer, severe heart failure and renal and hepatic dysfunction. The exclusion criteria for healthy individuals were: History of myocardial infarction, cerebrovascular accident, coronary bypass, coronary angiography with angioplasty or stenting or both or peripheral vascular disease were excluded. Informed consent was obtained from each patient and the study was approved by the Ethics Committee of the Affiliated Hospital of Qingdao University (Qingdao, China).

Cell culture. hVSMCs were purchased from Shanghai Jining Shiye Co., Ltd. (cat. no. JN-3270) and grown in DMEM (Gibco; Thermo Fisher Scientific, Inc.) supplemented with $10 \%$ fetal bovine serum (FBS; Gibco; Thermo Fisher Scientific, Inc.). Cells were then incubated at $37^{\circ} \mathrm{C}$ with $5 \% \mathrm{CO}_{2}$.

Cell transfection. The hVSMCs were first seeded into 6-well plates $\left(5 \times 10^{4}\right.$ cells/well $)$ and cultured at $37^{\circ} \mathrm{C}$ for $24 \mathrm{~h}$. Then, the hVSMCs were transfected with $1 \mu \mathrm{g}$ control-shRNA plasmid (cat. no. sc-108060; Santa Cruz Biotechnology, Inc.), $1 \mu \mathrm{g}$ MMP-9-shRNA plasmid (cat. no. sc-29400-SH, Santa Cruz Biotechnology, Inc.), $100 \mathrm{nM}$ mimic control (5'-CAG CUGGUUGAAGGGGACCAAA-3'; Shanghai GenePharma Co., Ltd.), 100 nM miR-133a-3p mimic (5'-UUUGGUCCC CUUCAACCAGCUG-3'; Shanghai GenePharma Co., Ltd.), or $100 \mathrm{nM}$ miR-133a-3p mimic $+1 \mu \mathrm{g}$ MMP-9-plasmid (cat. no. sc-400083-ACT, Santa Cruz Biotechnology, Inc.) using Lipofectamine ${ }^{\circledR} 2000$ (Invitrogen; Thermo Fisher Scientific, Inc.), according to the manufacturer's protocol. A total of $48 \mathrm{~h}$ after cell transfection, RT-qPCR was performed to determine the transfection efficiency.
$R T-q P C R$. Total RNA was extracted from blood, tissues or cells using the TRIzol Reagent (Thermo Fisher Scientific, Inc.) following the manufacturer's protocol. RT of extracted total RNA into cDNA was performed using the miScript Reverse Transcription kit (Qiagen $\mathrm{GmbH}$ ), according to the manufacturer's instructions. The temperature protocol for the reverse transcription reaction was as follows: $25^{\circ} \mathrm{C}$ for $5 \mathrm{~min}, 42^{\circ} \mathrm{C}$ for $60 \mathrm{~min}$ and $80^{\circ} \mathrm{C}$ for $2 \mathrm{~min}$. PCR was performed using the QuantiFast SYBR Green PCR kit (Qiagen GmbH). The amplification conditions were as follows: $10 \mathrm{~min}$ at $95^{\circ} \mathrm{C}$, followed by 35 cycles of $15 \mathrm{sec}$ at $95^{\circ} \mathrm{C}$ and $40 \mathrm{sec}$ at $55^{\circ} \mathrm{C}$. U6 or GAPDH was used as the internal reference. The primer sequences were as follows: U6 forward, 5'-GCTTCGGCAGCACATATACTA AAAT-3'; U6 reverse, 5'-CGCTTCACGAATTTGCGTGTC AT-3'; GAPDH forward, 5'-CTTTGGTATCGTGGAAGG ACTC-3'; GAPDH reverse, 5'-GTAGAGGCAGGGATGATG TTCT-3'; miR-133a-3p forward, 5'-CTTTAACCATTCTAG CTTTTCCAGGTA-3'; miR-133a-3p reverse, 5'-GACTTC GGCTGTGGACAAGATTAG-3'; MMP-9 forward, 5'-AGA CCTGGGCAGATTCCAAAC3'; MMP-9 reverse, 5'-CGG CAAGTCTTCCGAGTAGT-3'. The relative expression levels of genes were calculated using the $2^{-\Delta \Delta \mathrm{Cq}}$ method (21). All experiments were performed in triplicate.

Luciferase reporter assay. TargetScan version 7.2 (http://www.targetscan.org/vert_72/) was used to predict the potential targets of miR-133a-3p, and the binding sites between miR-133a and MMP-9. To confirm the association between miR-133a-3p and MMP-9, a dual-luciferase reporter assay was performed.

The wild-type and mutant 3'-UTR of MMP-9 (WT-MMP-9 and MUT-MMP-9, respectively) were cloned into a pmiR-RB-Report ${ }^{\mathrm{TM}}$ dual luciferase reporter gene plasmid vector (Guangzhou RiboBio Co., Ltd.) according to the manufacturer's protocol. hVSMCs were first seeded into 24 -well plates $\left(5 \times 10^{4}\right.$ cells per well) and then co-transfected with $100 \mathrm{ng}$ WT-MMP-9 or $100 \mathrm{ng}$ MUT-MMP-9 and $100 \mathrm{nM}$ miR-133a-3p mimic or $100 \mathrm{nM}$ mimic control using Lipofectamine ${ }^{\circledR} 2000$ (Invitrogen; Thermo Fisher Scientific, Inc.) in accordance with the manufacturer's protocol, together with Renilla luciferase pRL-TK vector (Promega Corporation) as a control. Following transfection for $48 \mathrm{~h}$, the relative luciferase activity was measured using the dual-luciferase reporter assay system (Promega Corporation), as per the manufacturer's protocol. All firefly luciferase activities were normalized to Renilla luciferase activity.

Cell counting kit-8 (CCK-8) assay. To determine cell proliferation, a CCK-8 assay was performed in accordance with the manufacturer's protocol (Sigma-Aldrich; Merck KGaA). hVSMCs were seeded into a 96 -well plate $\left(1 \times 10^{4}\right.$ cells/well $)$ and transfected with $1 \mu \mathrm{g}$ control-shRNA, $1 \mu \mathrm{g}$ MMP-9-shRNA, $100 \mathrm{nM}$ mimic control, $100 \mathrm{nM}$ miR-133a-3p mimic, or $100 \mathrm{nM}$ miR-133a-3p mimic + $1 \mu$ g MMP-9-plasmid for $48 \mathrm{~h}$ using Lipofectamine ${ }^{\circledR} 2000$ (Invitrogen; Thermo Fisher Scientific, Inc.), following the manufacturer's protocol. Subsequently, $10 \mu$ l CCK-8 solution (Sigma-Aldrich; Merck KGaA) was added and the cells were incubated for an additional $2 \mathrm{~h}$ at $37^{\circ} \mathrm{C}$ with $5 \% \mathrm{CO}_{2}$. Absorbance was detected at a wavelength of $490 \mathrm{~nm}$ using a micro-plate reader. 

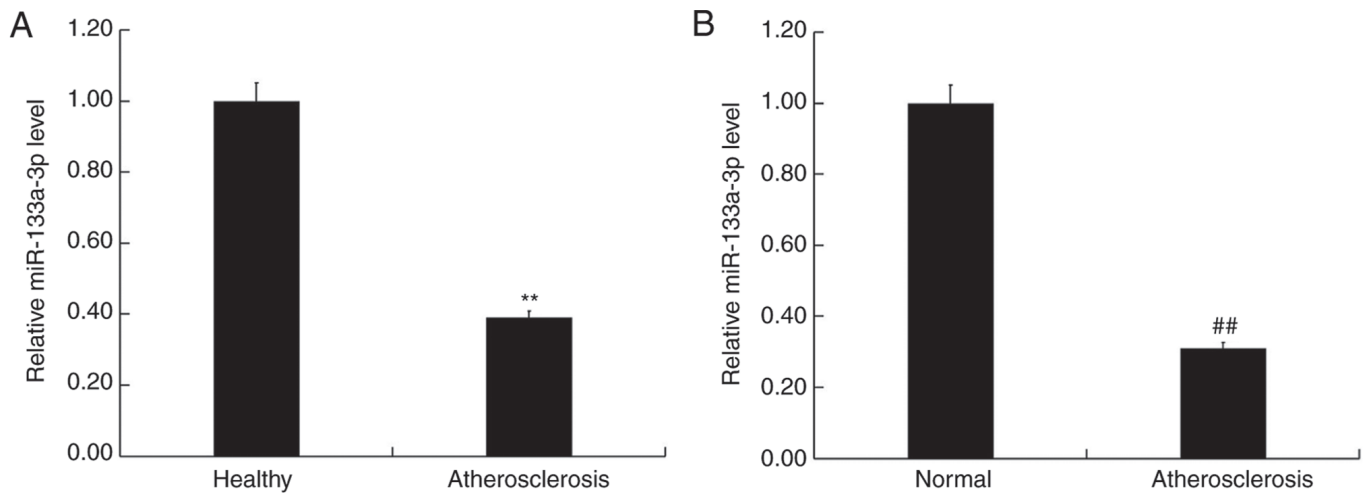

Figure 1. miR-133a-3p was downregulated in the blood and plaque tissue of patients with atherosclerosis. (A) RT-qPCR detected the relative mRNA expression of miR-133b in the blood of individuals with or without atherosclerosis. (B) RT-qPCR detected the relative mRNA expression of miR-133a-3p in the atherosclerotic vascular plaque tissues. Data are presented as the mean \pm standard deviation. ${ }^{* *} \mathrm{P}<0.01$ vs. healthy; ${ }^{\# \#} \mathrm{P}<0.01$ vs. normal. miR, miroRNA; RT-qPCR, reverse transcription-quantitative PCR. atherosclerosis, atherosclerotic plaque tissue; Normal, corresponding healthy vascular tissue.

Flow cytometry. Following cell transfection for $48 \mathrm{~h}$, the apoptotic rate of hVSMCs was determined using the Annexin V-fluorescein isothiocyanate/propidium iodide apoptosis detection kit [cat. no. 70-AP101-100; Hangzhou MultiSciences (Lianke) Biotech Co., Ltd.], according to the manufacturer's protocol. A flow cytometer was used to analyze cell apoptosis and data was analyzed using FlowJo software (version 7.6.1; FlowJo LLC).

Western blot analysis. Protein was extracted from blood, tissues or cells using radioimmunoprecipitation assay buffer (Beijing Solarbio Science \& Technology Co., Ltd.), according to the manufacturer's protocol. Total protein was quantified using a Bicinchoninic Acid protein assay kit (Beyotime Institute of Biotechnology). Equal quantities of protein (30 $\mu \mathrm{g}$ protein/lane) were separated via SDS-PAGE on a $10 \%$ gel and transferred to PVDF membranes. The membranes were blocked with 5\% skimmed milk at room temperature for $1.5 \mathrm{~h}$, followed by incubation with the following primary antibodies: MMP-9 (cat. no. 13667) and $\beta$-actin (cat. no. 4970; all 1:1,000; Cell Signaling Technology, Inc.) at $4^{\circ} \mathrm{C}$ overnight. Subsequently, membranes were incubated with an anti-rabbit IgG horseradish peroxidase-conjugated secondary antibody (1:2,000; cat. no. 7074; Cell Signaling Technology, Inc.) at room temperature for $2 \mathrm{~h}$. Protein bands were detected using the enhanced chemiluminescence detection system (Thermo Fisher Scientific, Inc.).

Statistical analyses. All experiments were performed at least three times. SPSS software version 17.0 (SPSS, Inc.,) was used for data analyses. Data were expressed as the mean \pm standard deviation. Differences between two groups were analyzed using a paired or unpaired Student's t-test, and comparisons between multiple groups were analyzed using one-way analysis of variance with Tukey's post hoc test. $\mathrm{P}<0.05$ was considered to indicate a statistically significant difference.

\section{Results}

Expression of miR-133a-3p in the blood and vascular plaque tissues of patients with AS. To determine the role of miR-133a-3p in AS, the level of miR-133a-3p expression was

\section{A WT-MMP-9 3'UTR 5'...UGUAAAUCCCCCACUGGGACCAAC... hsa-miR-133a-3p 3' GUCGACCAACUUCCCCUGGUUU MUT-MMP-9 $3^{\prime}$ UTR $5^{\prime} \ldots$... UGUAAAUCCCCACUGCCGAACCC...}

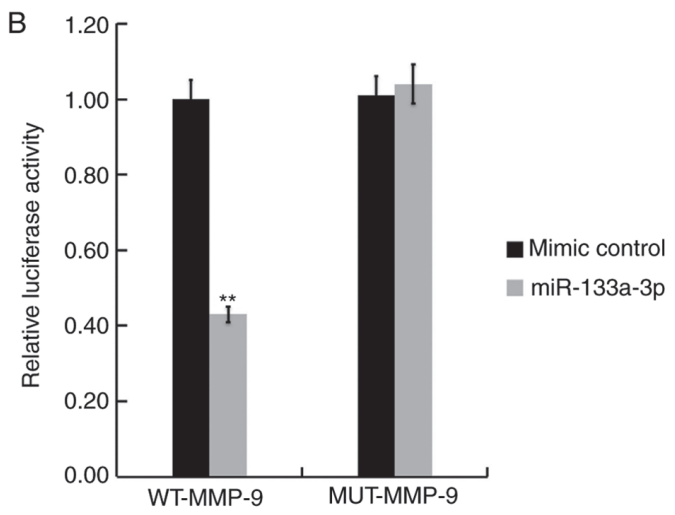

Figure 2. MMP-9 is a target gene of miR-133a-3p. (A) TargetScan predicted the binding site between the MMP-9 3'-UTR and miR-133a-3p. (B) miR-133a-3p mimics or mimic controls and WT or MUT MMP-9 3'UTR were co-transfected into human vascular smooth muscle cells. Luciferase activity was detected using dual-luciferase reporter assay. Data are presented as the mean \pm standard deviation. ${ }^{* *} \mathrm{P}<0.01$ vs. mimic control. MMP, matrix metalloprotease; miR, microRNA; UTR, untranslated region; WT, wild-type; MUT, mutant; has, homo sapiens.

detected in the blood and vascular plaque tissue of patients with or without AS using RT-qPCR. The results revealed that the expression of miR-133a-3p was significantly reduced in the blood of patients with AS compared with healthy controls (Fig. 1A). Furthermore, the expression of miR-133a-3p was significantly reduced in atherosclerotic plaque tissue compared with corresponding vascular tissue (Fig. 1B). These data indicated that downregulation of miR-133a-3p expression may be involved in the development of AS.

MMP-9 is a targetgene of miR-133a-3p. A search was performed using TargetScan to predict the target genes of miR-133a-3p. The results revealed the binding sites between miR-133a-3p and the 3'UTR of MMP-9 mRNA (Fig. 2A). Subsequently, a dual-luciferase reporter assay was performed to confirm whether miR-133a-3p interacts directly with the target gene, 

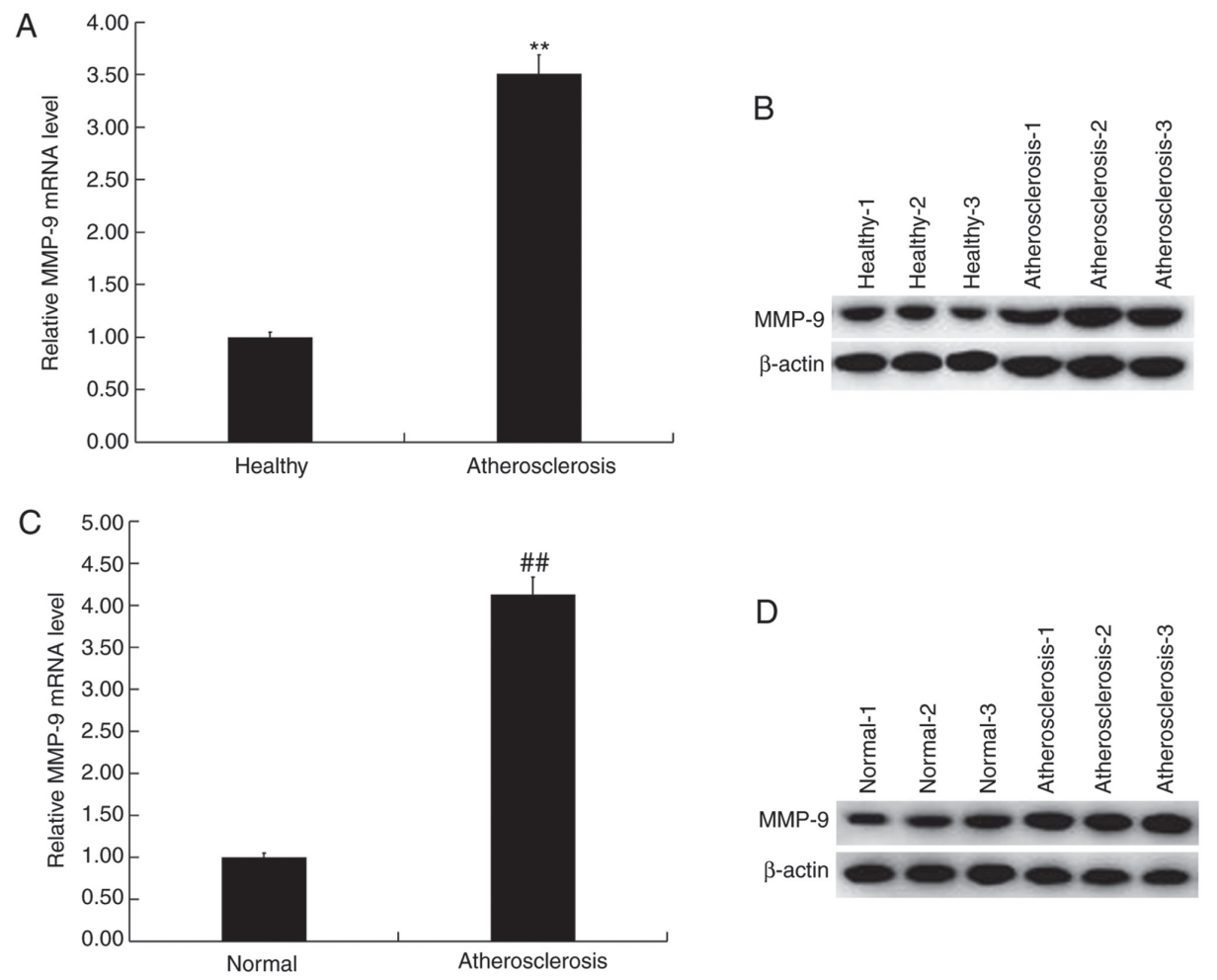

Figure 3. MMP-9 was upregulated in the blood and plaque tissue of patients with atherosclerosis. (A) RT-qPCR and (B) western blot analysis detected the relative mRNA and protein expression of MMP-9 in the blood (serum) of patients with or without atherosclerosis. (C) RTq-PCR and (D) western blot analysis detected the relative mRNA and protein expression of MMP-9 in atherosclerotic vascular plaque tissues. Data are presented as the mean \pm standard deviation. ${ }^{* *} \mathrm{P}<0.01$ vs. healthy; ${ }^{\# \#} \mathrm{P}<0.01$ vs. normal. MMP, matrix metalloprotease; RT-qPCR, reverse transcription-quantitative PCR; atherosclerosis, atherosclerotic vascular plaque tissue; Normal, corresponding healthy vascular tissue.

MMP-9 (Fig. 2B). The relative luciferase activity of hVSMCs co-transfected with WT-MMP-9 and miR-133a-3p mimic was significantly decreased compared with cells co-transfected with WT-MMP-9 and mimic control (Fig. 2B). No significant differences in relative luciferase activities were observed in hVSMCs co-transfected with MUT-MMP-9 and miR-133a-3p mimic and cells co-transfected with MUT-MMP-9 and mimic control (Fig. 2B). The results revealed that MMP-9 was a direct target gene of miR-133a-3p.

Expression of MMP-9 in the blood and vascular plaque tissue of patients with AS. The expression of MMP-9 in the blood and vascular plaque tissue of patients with or without AS were determined via RT-qPCR and western blotting. The results demonstrated a significant upregulation of the mRNA expression of MMP-9 in the blood of patients with AS compared with the healthy controls (Fig. 3A). Compared with healthy controls, the protein levels of MMP-9 in the blood of patients with AS markedly increased (Fig. 3B). Additionally, compared with the normal vascular tissue, the mRNA levels of MMP-9 were significantly upregulated in atherosclerotic plaque tissue (Fig. $3 \mathrm{C}$ ), whilst the protein levels of MMP-9 in atherosclerotic plaque tissues were also markedly higher compared with that in the normal vascular tissue (Fig. 3D).

Downregulation of MMP-9 inhibits hVSMC cell proliferation and induces apoptosis. hVSMCs were transfected with MMP-9-shRNA or control-shRNA for $48 \mathrm{~h}$. Transfection efficiency was then detected via RT-qPCR and western blot analysis. The results indicated a significantly decreased expression of MMP-9 in MMP-9-shRNA transfected hVSMCs at the mRNA level (Fig. 4A). Compared with the control group, MMP-9-shRNA transfection markedly reduced MMP-9 protein expression in hVSMCs (Fig. 4B). The role of MMP-9 in hVSMCs was determined using a CCK-8 assay (Fig. 4C) and flow cytometry (Fig. 4D). The results demonstrated the inhibition of proliferation and the induction of apoptosis upon MMP-9 downregulation.

Effect of miR-133a-3p on hVSMC proliferation and apoptosis. hVSMCs were transfected with the mimic control, miR-133a-3p mimic or miR-133a-3p mimic+MMP-9-plasmid for $48 \mathrm{~h}$. RT-qPCR revealed a significantly enhanced miR-133a-3p expression (Fig. 5A) in miR-133a-3p mimic-transfected hVSMCs. Compared with the control group, miR-133a-3p mimic transfection significantly reduced MMP-9 mRNA expression in hVSMCs, which was significantly reversed by MMP-9-plasmid (Fig. 5B). The protein levels of MMP-9 was also markedly reduced in VSMCs transfected with the miR-133a-3p mimic, which was reversed by transfection with the MMP-9-plasmid (Fig. 5C). The function of miR-133a-3p in hVSMC proliferation and apoptosis was subsequently investigated. The results of the CCK-8 assay demonstrated significant inhibition of proliferation (Fig. 5D) and induction of apoptosis (Fig. 5E) in miR-133a-3p mimic hVSMCs. These effects were reversed by the addition of the MMP-9-plasmid. 

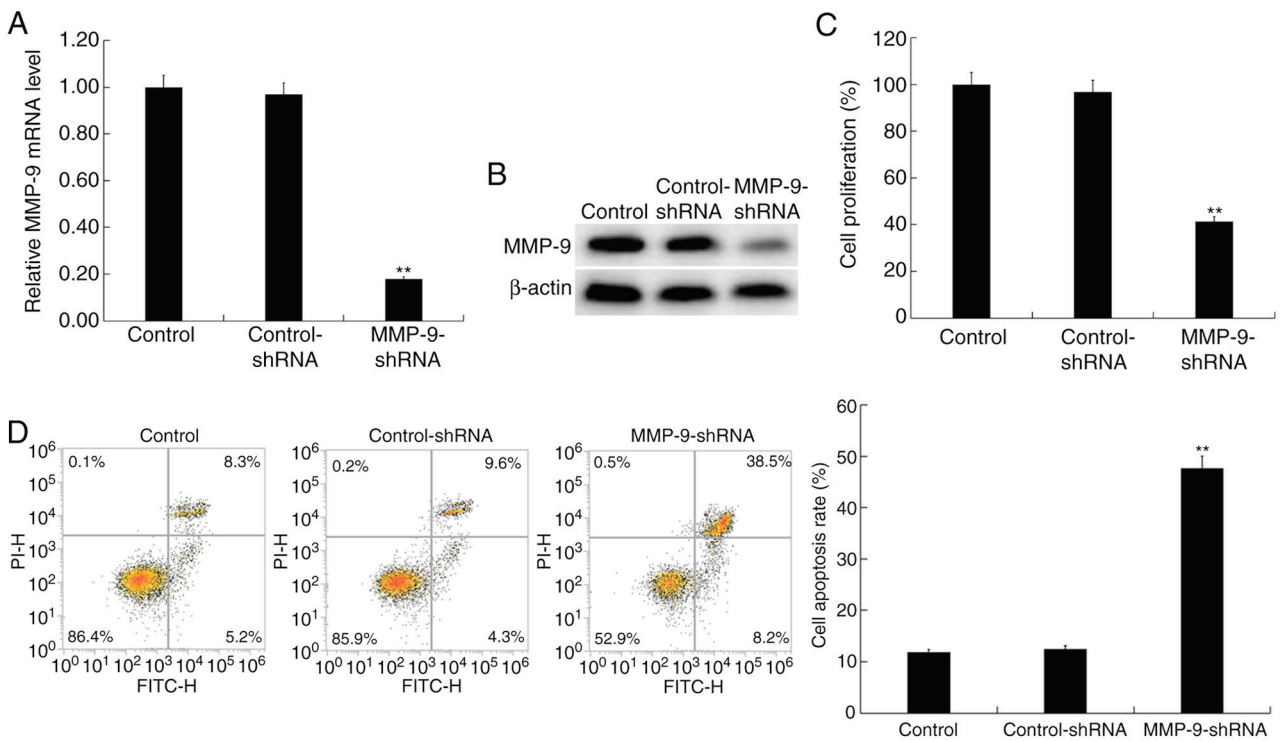

Figure 4. Effect of MMP-9 on hVSMCs. hVSMCs were transfected with control-shRNA and MMP-9 shRNA for 48 h. (A) RT-qPCR and (B) western blot analysis detected the mRNA and protein expression of MMP-9 in hVSMCs, respectively. A (C) Cell Counting kit-8 assay was performed to determine cell proliferation and (D) Flow cytometry was used to analyze cell apoptosis. Data are presented as the mean \pm standard deviation. ${ }^{* *} \mathrm{P}<0.01 \mathrm{vs}$. control. MMP, matrix metalloprotease; hVSMCs, human vascular smooth muscle cells; shRNA, short hairpin RNA; RT-qPCR, reverse transcription-quantitative PCR; PI, propidium iodide.
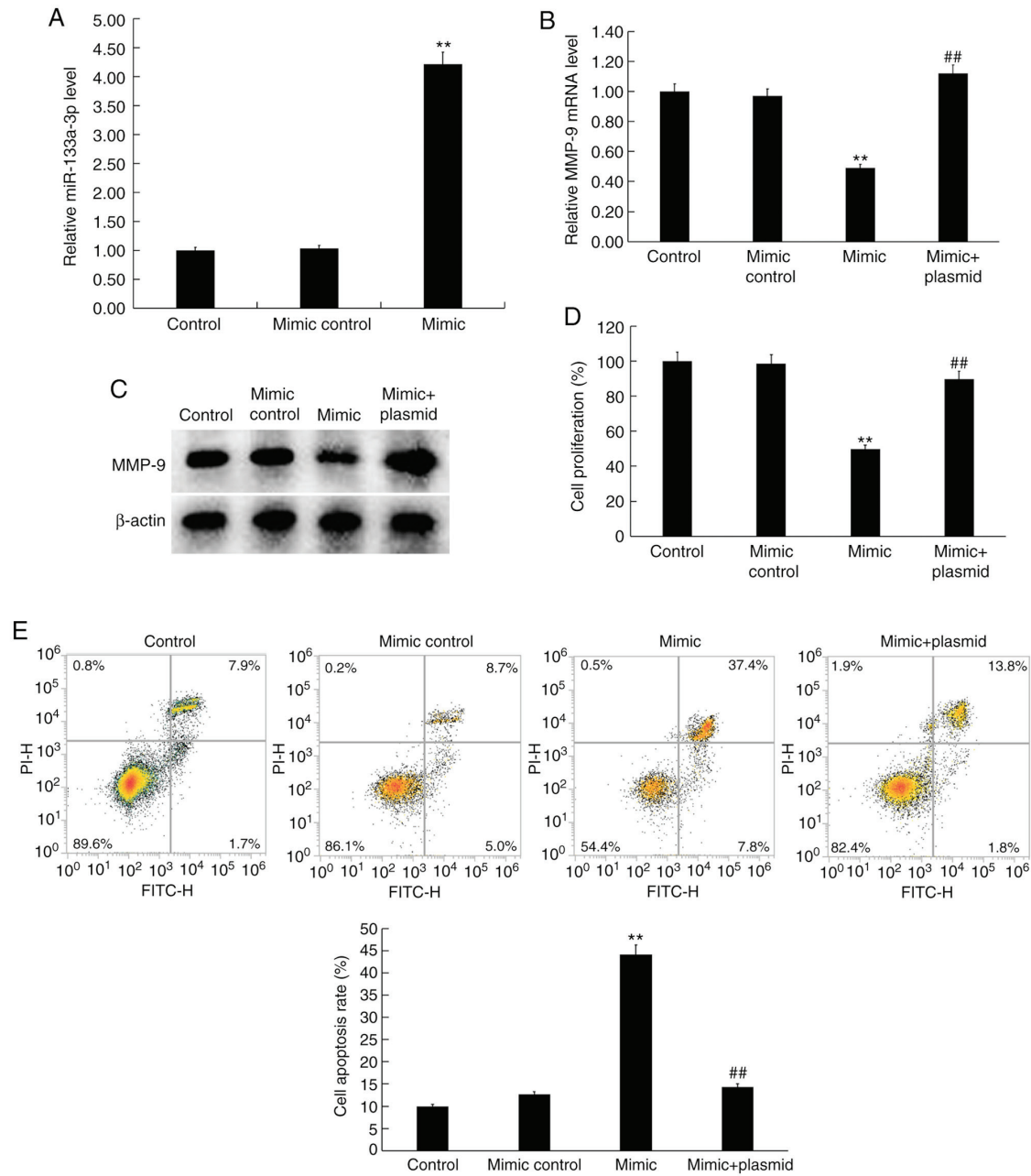

Figure 5. Effect of miR-133a-3p on hVSMCs. hVSMCs were transfected with mimic control, miR-133a-3p mimic or miR-133a-3p mimic+MMP-9 plasmid for $48 \mathrm{~h}$. (A) RT-qPCR detected levels of miR-133a-3p in hVSMCs. (B) RT-qPCR and (C) western blot analysis detected the mRNA and protein expressions of MMP-9 in hVSMCs, respectively. A (D) Cell Counting kit-8 assay was performed to determine cell proliferation and (E) Flow cytometry was used to detect cell apoptosis. Data are presented as the mean \pm standard deviation. ${ }^{* *} \mathrm{P}<0.01$ vs. control; ${ }^{\# /} \mathrm{P}<0.01$ vs. mimic. miR, microRNA; hVSMCs, human vascular smooth muscle cells; MMP, matrix metalloprotease; RT-qPCR, reverse transcription-quantitative PCR; PI, propidium iodide. 


\section{Discussion}

The present study demonstrated significant miR-133a-3p downregulation in the blood and atherosclerotic plaque tissue of patients with AS. MMP-9 was also revealed to be a direct target of miR-133a-3p, which was upregulated in the blood and atherosclerotic plaque tissues of patients with AS. Furthermore, the results determined that miR-133a-3p inhibited the proliferation of hVSMCs and induced apoptosis. These results indicated that miR-133a-3p may be a potential therapeutic target for the treatment of AS.

In recent years, different miRNAs have been identified to serve an important role in the development and progression of AS (10-13,22,23). The present study demonstrated a decreased expression of miR-133a-3p in the blood and atherosclerotic plaque tissue of individuals with or without AS. MMP-9 was identified as a target of miR-133a-3p, which was upregulated in AS. These data indicated the involvement of miR-133a-3p in the development of AS.

The proliferation and migration of VSMCs serve a crucial role in the formation and development of atherosclerotic plaques $(4,5)$. miRNAs have also been demonstrated to serve a role in modulating the function of VSMCs $(24,25)$. Thus, the current study assessed the in vitro effects of miR-133a-3p and MMP-9 on hVSMC.

MMP-9 serves an important role in the degradation of type IV and V collagen, and has been implicated in the pathogenesis of AS (26,27). MMP-9 has also been reported to serve a pivotal role in early $\mathrm{AS}$, vascular remodeling and the development of arterial plaque ruptures (28-31). In the present study, MMP-9 was revealed to be a direct target gene of miR-133a-3p and its low expression significantly inhibited the proliferation and promoted the cell apoptosis of hVSMCs. Furthermore, upregulation of miR-133a-3p via the miR-133a-3p mimic inhibited proliferation and promoted hVSMC cell apoptosis. Additionally, these effects were reversed by the addition of the MMP-9-plasmid.

In summary, the present study demonstrated that miR-133a-3p was downregulated in AS, which inhibited cell proliferation and induced apoptosis in hVSMCs by targeting MMP-9. Therefore, the miR-133a-3p/MMP-9 axis may be a potential target for the treatment of AS.

However, the present study is only a preliminary investigation into the role of miR-133a-3p in AS and as such, further studies are required. For example, the expression of miR-133a-3p and MMP-9 and their association with AS severity should be determined. The expression of miR-133a-3p and MMP-9 in blood and their association with that in vascular plaque tissue should also be further studied. Additionally, the association between MMP-9 and miR-133a-3p in patients with AS requires further assessment. Finally, an in vivo study of miR-133a-3p in AS should be performed in future studies.

\section{Acknowledgements}

The authors would like to thank Mrs. Xiaohui Zhang, Department of Emergency Internal Medicine, The Affiliated Hospital of Qingdao University (Qingdao, China), for assisting with sample collection and statistical analyses. The authors would also like to acknowledge Director Dahai Li of the
Central Laboratory (the Affiliated Hospital of Qingdao University, Qingdao, China) for experimental guidance and quality control.

\section{Funding}

No funding was received.

\section{Availability of data and materials}

All datasets used and/or analyzed during the current study are available from the corresponding author on reasonable request.

\section{Authors' contributions}

LS contributed to study design, data collection, data interpretation and manuscript preparation; $\mathrm{CY}$ and XT contributed to data collection, statistical analysis and data interpretation; CM and LW contributed to statistical analysis and data interpretation; DX, CC and XC contributed to manuscript preparation and statistical analysis; TJ, YG and ZL contributed to data interpretation and literature search; SC contributed to study design and funds collection. All authors read and approved the final manuscript.

\section{Ethics approval and consent to participate}

Informed consent was obtained from each patient and the study was approved by the Ethics Committee of the Affiliated Hospital of Qingdao University (Qingdao, China).

\section{Patient consent for publication}

All patients agreed to publication.

\section{Competing interests}

The authors declare that they have no competing interests.

\section{References}

1. Pant S, Deshmukh A, Gurumurthy GS, Pothineni NV, Watts TE, Romeo F and Mehta JL: Inflammation and atherosclerosisrevisited. J Cardiovasc Pharmacol Ther 19: 170-178, 2014.

2. Weber $\mathrm{C}$ and Noels $\mathrm{H}$ : Atherosclerosis: Current pathogenesis and therapeutic options. Nat Med 17: 1410-1422, 2011.

3. Eelen G, de Zeeuw P, Simons M and Carmeliet P: Endothelial cell metabolism in normal and diseased vasculature. Circ Res 116: 1231-1244, 2015.

4. Rudijanto A: The role of vascular smooth cells on the pathogenesis of atherosclerosis. Acta Med Indones 39: 86-93, 2007.

5. Cersosimo E and DeFronzo RA: Insulin resistance and endothelial dysfunction: The road map to cardiovascular diseases. Diabetes Metab Res Rev 22: 423-436, 2006.

6. Bartel DP: MicroRNAs: Genomics, biogenesis, mechanism, and function. Cell 116: 281-297, 2004.

7. Ebert MS and Sharp PA: Roles for microRNAs in conferring robustness to biological processes. Cell 149: 515-524, 2012.

8. Rogers $\mathrm{K}$ and Chen X: Biogenesis, turnover, and mode of action of plant microRNAs. Plant Cell 25: 2383-2399, 2013.

9. Hayes J, Peruzzi PP and Lawler S: MicroRNAs in cancer: Biomarkers, functions and therapy. Trends Mol Med 20: 460-469, 2014.

10. Qu Y and Zhang N: miR-365b-3p inhibits the cell proliferation and migration of human coronary artery smooth muscle cells by directly targeting ADAMTS1 in coronary atherosclerosis. Exp Ther Med 16: 4239-4245, 2018. 
11. Wu CY, Zhou ZF, Wang B, Ke ZP, Ge ZC and Zhang XJ: MicroRNA-328 ameliorates oxidized low-density lipoprotein-induced endothelial cells injury through targeting HMGB1 in atherosclerosis. J Cell Biochem: Oct 15, 2018 (Epub ahead of print).

12. de Ronde MWJ, Kok MGM, Moerland PD, Van den Bossche J, Neele AE, Halliani A, van der Made I, de Winther MPJ, Meijers JCM, Creemers EE and Pinto-Sietsma SJ: High miR-124-3p expression identifies smoking individuals susceptible to atherosclerosis. Atherosclerosis 263: 377-384, 2017.

13. Chen Z, Wang M, Huang K, He Q, Li H and Chang G: MicroRNA-125b affects vascular smooth muscle cell function by targeting serum response factor. Cell Physiol Biochem 46 1566-1580, 2018.

14. House R, Majumder M, Janakiraman H, Ogretmen B, Kato M, Erkul E, Hill E, Atkinson C, Barth J, Day TA and Palanisamy V: Smoking-induced control of miR-133a-3p alters the expression of EGFR and HuR in HPV-infected oropharyngeal cancer. PLoS One 13: e0205077, 2018.

15. Tang Y, Pan J, Huang S, Peng X, Zou X, Luo Y, Ren D, Zhang X, Li R, He P and Wa Q: Downregulation of miR-133a-3p promotes prostate cancer bone metastasis via activating PI3K/AKT signaling. J Exp Clin Cancer Res 37: 160, 2018.

16. Zhang Y, Dun Y, Zhou S and Huang XH: LncRNA HOXD-AS1 promotes epithelial ovarian cancer cells proliferation and invasion by targeting miR-133a-3p and activating Wnt/3-catenin signaling pathway. Biomed Pharmacother 96: 1216-1221, 2017.

17. Gao L, Li SH, Tian YX, Zhu QQ, Chen G, Pang YY and Hu XH: Role of downregulated miR-133a-3p expression in bladder cancer: A bioinformatics study. Onco Targets Ther 10: 3667-3683, 2017.

18. Van den Steen PE, Dubois B, Nelissen I, Rudd PM, Dwek RA and Opdenakker G: Biochemistry and molecular biology of gelatinase B or matrix metalloproteinase-9(MMP-9). Crit Rev Biochem Mol Biol 37: 375-536, 2002.

19. Hobeika MJ, Thompson RW, Muhs BE, Brooks PC and Gagne PJ: Matrix metalloproteinases in peripheral vascular disease. J Vasc Surg 45: 849-857, 2007

20. Shin SS, Ko MC, Noh DH, Hwang B, Park Y, Park SL, Kim WJ and Moon S: Morin inhibits PDGF-induced proliferation, migration, and invasion of vascular smooth muscle cells via modulating p27KIP1, AKT, and MMP-9 activities. Gen Physiol Biophys 37: 633-645, 2018.
21. Livak KJ and Schmittgen TD: Analysis of relative gene expression data using real-time quantitative PCR and the 2(-Delta Delta C(T)) method. Methods 25: 402-408, 2001.

22. Lu Y, Thavarajah T, Gu W, Cai J and Xu Q: Impact of miRNA in Atherosclerosis. Arterioscler Thromb Vasc Biol 38: e159-e170, 2018.

23. Feinberg MW and Moore KJ: MicroRNA regulation of atherosclerosis. Circ Res 118: 703-720, 2016.

24. Sun QR, Zhang X and Fang K: Phenotype of vascular smooth muscle cells (VSMCs) is regulated by miR-29b by targeting sirtuin 1. Med Sci Monit 24: 6599-6607, 2018.

25. Li K, Pan J, Wang J, Liu F and Wang L: miR-665 regulates VSMCs proliferation via targeting FGF9 and MEF2D and modulating activities of Wnt/ $\beta$-catenin signaling. Am J Transl Res 9: 4402-4414, 2017.

26. Szmitko PE, Wang CH, Weisel RD, Jeffries GA, Anderson TJ and Verma S: Biomarkers of vascular disease linking inflammation to endothelial activation: Part II. Circulation 108: 2041-2048, 2003.

27. Florence JM, Krupa A, Booshehri LM, Allen TC and Kurdowska AK: Metalloproteinase-9 contributes to endothelial dysfunction in atherosclerosis via protease activated receptor-1. PLoS One 12: e0171427, 2017.

28. Newby AC: Metalloproteinase expression in monocytes and macrophages and its relationship to atherosclerotic plaque instability. Arterioscler Thromb Vasc Biol 28: 2108-2114, 2008.

29. Bendeck MP, Conte M, Zhang M, Nili N, Strauss BH and Farwell SM: Doxycycline modulates smooth muscle cell growth, migration, and matrix remodeling after arterial injury. Am J Pathol 160: 1089-1095, 2002.

30. Kunz J: Matrix metalloproteinases and atherogenesis in dependence of age. Gerontology 53: 63-73, 2007.

31. Newby AC: Dual role of matrix metalloproteinases (matrixins) in intimal thickening and atherosclerotic plaque rupture. Physiol Rev 85: 1-31, 2005.

This work is licensed under a Creative Commons Attribution-NonCommercial-NoDerivatives 4.0 International (CC BY-NC-ND 4.0) License. 